\title{
A IMPORTÂNCIA DOS ASPECTOS PSICOLÓGICOS NA REABILITAÇÃO DO PACIENTE COM DOENÇA ARTERIAL CORONARIANA
}

DOI: 10.22289/2446-922X.V7N2A9

\author{
Jéssica Helena Souza de França ${ }^{1}$ \\ Yasmin Nascimento Passos
}

\section{RESUMO}

As doenças cardíacas representam um rompimento da vida do paciente em sua rotina diária, acarretando problemas emocionais, que por vezes podem dificultar 0 tratamento. $O$ desencadeamento da doença arterial coronariana em um membro familiar pode ser fonte de estresse para todos os membros da família. Portanto, por ser uma doença que traz ao indivíduo não apenas sofrimento físico, mas também psicológico, se faz necessária a reabilitação, tendo em vista o benefício que esta trará na vida desses pacientes. O presente trabalho tem como objetivo realizar uma revisão narrativa da literatura sobre a importância dos aspectos psicológicos na reabilitação do paciente com doença arterial coronariana. O método escolhido foi uma pesquisa bibliográfica dos últimos 25 anos em livros e periódicos online específicos no tema. A partir do material selecionado, foram elaboradas três categorias. A primeira categoria sobre a doença arterial coronariana aborda a definição de doença arterial coronariana, suas manifestações clínicas, fatores de risco, diagnóstico, formas de tratamento e reabilitação. $\mathrm{Na}$ segunda categoria serão descritos os aspectos psicológicos presentes na reabilitação, e na terceira e ultima categoria será descrito o papel do psicólogo na reabilitação. Como resultado, observou-se que os aspectos psicológicos são de suma relevância no processo de reabilitação do paciente com doença arterial coronariana, pois exercem influência no desenvolvimento da doença, interferindo no prognóstico e na adesão do paciente ao processo de tratamento e reabilitação.

Palavras-chave: Doenças cardiovasculares; Família; Psicólogo da saúde; Psicólogo na reabilitação.

\section{THE IMPORTANCE OF PSYCHOLOGICAL ASPECTS IN THE REHABILITATION OF THE PATIENT WITH CORONARY ARTERIAL DISEASE}

\section{ABSTRACT}

Heart diseases represent a disruption of the patient's life in his daily routine, resulting in emotional problems, which can sometimes hinder treatment. The onset of coronary artery disease in a family member can be a source of stress for all family members. Therefore, because it is a disease that brings to the individual not only physical suffering, but also psychological suffering, rehabilitation is necessary, considering the benefits that this will bring in

\footnotetext{
${ }^{1}$ Endereço eletrônico de contato: jessyfranca@yahoo.com

Recebido em 29/05/2021. Aprovado pelo conselho editorial para publicação em 03/08/2021.
}

Rev. Psicol Saúde e Debate. Ago., 2021:7(2): 131-150. 
the lives of these patients. The present work aims to conduct a narrative literature review about the importance of psychological aspects in the rehabilitation of the patient with coronary artery disease. The method chosen was a bibliographic research of the last 25 years in books and online journals specific to the theme. From the selected material, three categories were elaborated. The first category about coronary artery disease addresses the definition of coronary artery disease, its clinical manifestations, risk factors, diagnosis, forms of treatment and rehabilitation. In the second category, the psychological aspects present in rehabilitation, will be described, and in the third and last category, the role of the psychologist in rehabilitation will be described. As a result, it was observed that the psychological aspects are of utmost relevance in the rehabilitation process of the patient with coronary artery disease, as they influence the development of the disease, interfering in the prognosis and in the patient adherence to the treatment and rehabilitation process.

Keywords: Cardiovascular diseases; Family; Health psychologist; Rehabilitation psychologist.

\section{LA IMPORTANCIA DE LOS ASPECTOS PSICOLÓGICOS EM LA REHABILITACIÓN DEL PACIENTE CON ENFERMEDAD ARTERIAL CORONARIA}

\section{RESUMEN}

La enfermedad cardíaca representa una interrupción de la vida del paciente en su rutina diaria, provocando problemas emocionales, que en ocasiones pueden dificultar el tratamiento. La aparición de la enfermedad de las arterias coronarias en una persona puede ser una fuente de estrés para todos los miembros de la familia. A qué es una enfermedad que trae al individuo no solo sufrimiento físico sino también psicológico, la rehabilitación es necesaria en vista del beneficio que traerá en la vida de estos pacientes. Este trabajo tiene como objetivo realizar una revisión de literatura narrativa sobre la importancia de los aspectos psicológicos en la rehabilitación de pacientes con enfermedad arterial coronaria. El método elegido consistió en una búsqueda bibliográfica de los últimos veinticinco años en libros y revistas electrónicas específicos del tema. A partir del material selecionado, se elaboraron tres categorias. La primera categoría acerca de la enfermedad de las arterias coronarias aborda la definición de enfermedad de las arterias coronarias, sus sintomas clínicos, factores de riesgo, diagnóstico, formas de tratamiento y rehabilitación. En la segunda categoría fueron descritos los aspectos psicológicos presentes en la rehabilitación, y en la tercera y última categoría se describe el papel del psicólogo en la rehabilitación. Como resultado, se observó que los aspectos psicológicos son de gran importancia en el proceso de rehabilitación de los pacientes con enfermedad coronaria, ya que influyen en el desarrollo de la enfermedad, interfiriendo con el pronóstico y la adherencia del paciente al tratamiento y rehabilitación.

Palabras clave: Enfermedades cardiovasculares; Familia; Psicólogo de la salud; Psicólogo en la rehabilitación. 


\section{INTRODUÇÃO}

As doenças cardiovasculares constituem um importante problema de saúde pública em todo o mundo, representando altos custos em assistência médica (Escudeiro \& Melo, 2010). Elas são responsáveis por cerca de 29,4\% dos óbitos, e dentre as de maior ocorrência destacam-se: $O$ acidente vascular encefálico, a doença arterial coronariana e a hipertensão arterial sistêmica, sendo causadas por fatores de risco bem estabelecidos (Lamas, 2010). Essas doenças aparecem em primeiro lugar na mortalidade no Brasil, sendo responsáveis por quase um terço dos óbitos, com 65\% das mortes na faixa etária de 30 a 69 anos de idade (Oliveira et al., 2015).

O desencadeamento da doença arterial coronariana em um membro familiar pode ser fonte de estresse para todos os membros da família. A descoberta de uma doença crônica, principalmente quando atinge adultos que desempenham papéis sociais definidos, pode gerar desequilíbrio no sistema familiar, o que pode exigir rearranjos. O cônjuge, os filhos do paciente ou mesmo outros membros familiares, passam a assumir atividades que eram desempenhadas pelo paciente antes da internação, assim, a doença tende a afetar a estrutura familiar nos aspectos afetivos e socioeconômicos, principalmente naquelas famílias onde o paciente era o provedor financeiro (Dantas et al., 2002).

De acordo com Romano (1998), as doenças cardíacas representam um rompimento da vida do paciente em sua rotina diária, acarretando problemas emocionais, que por vezes podem dificultar o tratamento. Portanto, por ser uma doença que traz ao indivíduo não apenas sofrimento físico, mas também psicológico, e por exigir deste, mudanças repentinas em diversos aspectos de sua vida, como nas relações amorosas, trabalho, atividades físicas, relações sexuais, relações sociais, entre outros, se faz necessária a reabilitação, tendo em vista o benefício que esta trará na vida desses pacientes.

A reabilitação é um processo contínuo de uma equipe multiprofissional que atua com o objetivo de ajudar o paciente a realizar suas potencialidades físicas, sociais, psíquicas e laborais, para que possa ter uma maior independência, autonomia e qualidade de vida (Angerami, 2004).

A reabilitação geralmente acontece no hospital quando a equipe de saúde solicita o psicólogo, ou ele através de busca ativa realiza uma avaliação psicológica para verificar se o paciente apresenta demanda psicológica, como por exemplo, Rev. Psicol Saúde e Debate. Ago., 2021:7(2): 131-150. 
sintomas de ansiedade, depressão, irritabilidade etc. Caso o paciente apresente demanda, o psicólogo fará o acompanhamento deste paciente durante todo o seu processo de hospitalização, se caracterizando assim o processo de reabilitação psicológica. A atuação do psicólogo é fundamental no acompanhamento do paciente com doença arterial coronariana, desde o pré-operatório até a sua alta, podendo ser estendida ao domicilio quando há possibilidade de uma equipe multidisciplinar (home care) com o psicólogo inserido nesta equipe (grifo nosso).

O psicólogo no processo de reabilitação tem uma função muito importante, pois é através da comunicação que o mesmo irá favorecer ao paciente e aos familiares o enfrentamento da situação de adoecimento. O psicólogo exerce inúmeras atividades, como auxílio ao enfrentamento da doença e de seu tratamento, observação e apoio aos transtornos mentais associados à doença, fazendo com que o paciente e sua família se tornem ativos neste processo (Angerami, 2004).

A reabilitação permitirá ao paciente se adaptar a um novo estilo de vida, mostrando que a condição imposta pela doença não implicará na impossibilidade de realizar atividades, pois estas serão adaptadas aos seus limites e possibilidades. Ela abrange inúmeras dimensões da vida do sujeito, envolvendo-o por inteiro. Como na maioria das vezes a cirurgia é o único recurso de tratamento para a doença arterial coronariana, o apoio psicológico em reabilitação é fundamental, desde o início do tratamento, antes mesmo da cirurgia e após esta (Eickhoff et al., 2014).

O presente trabalho tem como objetivo fazer uma revisão narrativa da literatura que aborde a importância dos aspectos psicológicos na reabilitação do paciente com doença arterial coronariana. $O$ interesse pela escolha do tema ocorreu a partir da prática no hospital como Psicólogas atuando junto a alguns pacientes internados com doença arterial coronariana.

\section{MÉTODO}

A metodologia escolhida foi uma pesquisa bibliográfica dos últimos 25 anos. Foram utilizados 3 livros e 26 artigos científicos. Foi feita uma leitura exploratória dos 3 livros e estavam de acordo com os critérios de inclusão. Os livros são referência na área da psicologia hospitalar, abordam a temática proposta pelo trabalho e por isso foram importantes para a sua construção. 
O primeiro livro do autor Romano (1998), foi usado para falar sobre as mudanças ocorridas durante o surgimento de uma doença cardíaca. O segundo livro do autor Angerami (2004), foi utilizado para falar sobre o psicólogo no processo de reabilitação do paciente e sua atuação na equipe multiprofissional. O terceiro e último livro do autor Simonetti (2011) foi usado para falar de aspectos psicológicos em torno do adoecimento.

Os artigos científicos foram buscados nos bancos de dados online: Scientific Electronic Library Online (SCIELO), Periódicos Eletrônicos de Psicologia (PEPSIC), Centro Universitário de Maringá (CESUMAR), Instituto Superior de Psicologia Aplicada (ISPA), Universidade Católica de Petrópolis (UCP), Universidade Federal da Bahia (UFBA), Universidade Regional do Noroeste do Rio Grande do Sul (UNIJUÍ), Universidade Paulista (UNIP), Universidade de São Paulo (USP), Arquivos Brasileiros de Cardiologia, Sociedade de Cardiologia do Rio de Janeiro (SOCERJ), Sociedade Brasileira de Psicologia Hospitalar (SBPH) e banco de dissertações da Universidade Federal do Rio de Janeiro (UFRJ).

Foi feita uma leitura exploratória de 26 artigos científicos que estavam dentro do período dos últimos 25 anos e foram considerados como critérios de inclusão também, aqueles que atendiam aos conceitos dos descritores: Cardiopatias, doenças cardiovasculares, doença arterial coronariana, reabilitação, aspectos psicológicos, psicólogo no hospital e psicólogo na reabilitação. Aqueles que não atendiam a estes conceitos e ao período citado anteriormente foram excluídos. Os resultados foram apresentados de forma descritiva, divididos em categorias com a temática proposta.

A partir da revisão da literatura foram elaboradas três categorias que possibilitaram a análise do tema proposto. As 3 categorias seguem a seguinte ordem: Doença arterial coronariana, onde será descrita a definição de doença arterial coronariana, suas manifestações clínicas, os fatores de risco, o diagnóstico, as formas de tratamento e reabilitação. Na segunda categoria são descritos os aspectos psicológicos presentes na reabilitação. Na terceira e última categoria será descrito o papel do psicólogo no processo de reabilitação.

\section{DOENÇA ARTERIAL CORONARIANA}




\section{Definição de doença arterial coronariana}

A doença arterial coronariana é o resultado da formação de placas de aterosclerose, que são placas de tecido fibroso e colesterol, que crescem e se acumulam na parede dos vasos a ponto de dificultar ou até mesmo impedir a passagem do sangue. Esta lesão pode ser acelerada por fumo, pressão alta, colesterol sanguíneo elevado e diabetes (Sociedade de Cardiologia do Estado do Rio de Janeiro, [SOCERJ] 2013).

Quando o entupimento da artéria por aterosclerose envolve mais de 50 a $70 \%$ do seu diâmetro, o fluxo sanguíneo se torna insuficiente para nutrir a porção do coração irrigada pela artéria doente, especialmente quando a necessidade de oxigênio é maior, como por exemplo, durante um exercício físico. A irrigação inadequada de uma determinada região denomina-se isquemia. Quando prolongada, a isquemia pode provocar a morte do tecido, a este fenômeno chamamos de infarto. Quando isto ocorre o termo usado é isquemia miocárdica e infarto do miocárdio. Ambas as situações são percebidas pelo paciente como dor no peito (SOCERJ, 2013).

\section{Manifestações clínicas}

De acordo com Cantus e Ruiz (2011) a doença arterial coronariana, pode se desenvolver ao longo de vários anos, os sintomas podem não ser frequentemente sentidos, até que os bloqueios sejam graves e ameaçadores à vida. Os seus sintomas diferem de pessoa para pessoa, mas os sintomas típicos incluem: Desconforto ou dor intensa no peito, denominado de "angina", falta de ar, fadiga extrema devido esforço físico, inchaço nos pés, enxaqueca, dor nos ombros ou braços, dor na mandíbula, náuseas e vômitos. As mulheres podem ter dores atípicas no peito, podendo ser passageiras ou agudas, e percebidas no abdômen, nas costas ou nos braços.

\section{Fatores de risco}

Entre os principais fatores de risco estão à história familiar de doença arterial coronariana, hipertensão arterial, diabetes mellitus, tabagismo, dislipidemia (níveis elevados de gordura no sangue), obesidade e sedentarismo. As pessoas que apresentam algum desses fatores de risco estão mais propensas para desenvolver a doença (Gus et al., 2002). De acordo com Maia et al. (2007), a hipertensão arterial é a maior representante dos fatores de risco cardiovasculares. 
Em países da América Latina, a presença de fatores psicossociais também está associada com risco aumentado de infarto agudo do miocárdio. Estresse emocional relacionado a ambiente de trabalho, problemas pessoais e familiares também representam risco para adoecer (Jurkiewicz \& Romano, 2009).

Evidências clínicas também mostraram uma estreita relação entre a doença arterial coronariana e a depressão. A depressão está associada a certas cardiopatias, há evidências de maior prevalência da depressão entre os portadores da doença arterial coronariana (Jurkiewicz \& Romano, 2009). Pela doença também estar relacionada com sintomas psicológicos se faz necessário o apoio psicológico com esses pacientes (Grifo nosso).

\section{Diagnóstico}

Para diagnosticar esta doença, é necessária a realização de exames. O eletrocardiograma comum ou em repouso é um registro de atividades elétricas do coração que fornece várias informações, inclusive a presença de isquemia. No entanto, o paciente pode ter obstruções arteriais e exibir um eletrocardiograma normal, pois em repouso, as obstruções existentes permitem a passagem de uma quantidade de sangue suficiente para irrigar aquele músculo que não está sendo exigido por um esforço maior (SOCERJ, 2013).

O teste de esforço ou ergometria é uma forma de tentar compensar a deficiência do eletrocardiograma em repouso. Neste teste, o paciente se exercita numa esteira ou bicicleta ergométrica, enquanto o eletrocardiograma, o pulso e a pressão arterial são registrados, de modo a sobrecarregar o coração e tentar evidenciar algum sinal de isquemia (SOCERJ, 2013).

O cateterismo cardíaco com cinecoronariografia significa introduzir um pequeno tubo flexível chamado de "cateter" através do braço ou da virilha sob anestesia local, avançando até o coração e artérias coronárias, onde são feitas injeções de contraste radiológico. Assim, é possível visualizar através de raios- $X$ as artérias e localizar a forma, a extensão e a gravidade das obstruções encontradas. Este método na maioria das vezes não é capaz por si só de definir a conduta a ser tomada em relação ao paciente, sendo necessária uma avaliação clínica (SOCERJ, 2013).

\section{Formas de tratamento e reabilitação}


São três os objetivos do tratamento medicamentoso: Diminuir o trabalho cardíaco, melhorar a oferta de oxigênio e diminuir a coagulabilidade do sangue (SOCERJ, 2013). De acordo com Pêgo-Fernandes et al. (2008), o tratamento clínico realizado através de medicamentos e mudanças nos hábitos de vida, apresenta bons resultados, entretanto quando há angina refratária (diminuição da força de concentração do coração) que ocasiona insuficiência cardíaca e risco de vida, faz-se necessária a realização da cirurgia.

A cirurgia cardíaca oferece a técnica cirúrgica de revascularização do miocárdio, a qual permite que o sangue seja transportado para o território isquêmico através de um conduto. A revascularização do miocárdio tem como principal objetivo reestabelecer o fornecimento de sangue adequado ao miocárdio (músculo que possui alta demanda de oxigênio e nutrientes), e para isso abordam as artérias coronárias, que são o sistema de condução do sangue (Pêgo-Fernandes et al., 2008).

A cirurgia de revascularização do miocárdio está indicada para aqueles pacientes em que o tratamento clínico não consegue controlar a angina (dor intensa no peito) ou para aqueles que possuem um elevado grau de obstrução das principais artérias coronárias, levando ao risco de vida. Geralmente são pacientes que apresentam lesões coronarianas difusas, com acometimento de mais de um território coronariano e com envolvimento de artérias vitais (Pêgo-Fernandes et al., 2008). Após a cirurgia, o paciente precisará aderir ao processo de reabilitação (Faro, 2006).

A reabilitação abrange diversas dimensões, sendo entendida como um processo que está longe de ser apenas a recuperação de funções perdidas ou alteradas. $\mathrm{Na}$ área da saúde, é uma especialidade que aborda o indivíduo em constante convívio com o seu ambiente social. Os principais objetivos da reabilitação é promover a implicação do paciente e da sua família no planejamento e na instalação de cuidados, que pretendem proporcionar maior independência para o paciente (Faro, 2006).

Na reabilitação é necessário que o paciente tenha todo o apoio dos profissionais envolvidos e dos seus familiares, pois são utilizadas técnicas e ações interdisciplinares que precisam ter como meta comum a melhora das funções diminuídas ou perdidas e 0 cuidado com o sujeito, preservando sua autonomia para realizar atividades (Faro, 2006).

O início do processo de reabilitação é o momento mais delicado para o paciente, para a família e para os profissionais que atuam junto a eles, pois é quando começam 
a conhecer as limitações provocadas pela doença, que podem exigir rearranjos em sua rotina diária, o que pode também gerar negação, dúvidas e incertezas em relação ao tratamento. Por isso, na reabilitação há a retomada tanto das funções orgânicas quanto dos aspectos psíquicos e sociais (Faro, 2006).

A reabilitação, entendida como sendo um processo, possui início e deverá ter meio e fim, pois o paciente e a família precisam receber as orientações e intervenções necessárias até o momento em que se considerem capazes de lidar com as demandas, sozinhos, e que os profissionais percebam que eles realmente mostram-se aptos para exercer suas atividades. Também deve haver o reconhecimento de que o melhor nível de recuperação ocorrerá em função do estilo de vida do paciente e de suas condições clínicas (Faro, 2006).

O processo de reabilitação não tem como objetivo a cura, ela abrange outros aspectos além dos físicos, que são os psíquicos, sociais, culturais, econômicos, políticos, entre outros. Ela propõe o desenvolvimento de conhecimentos, comportamentos e habilidades que façam com que os indivíduos envolvidos nesse processo, possam ter autonomia no desempenho das suas atividades, estimulando a sua melhora funcional (melhores condições física, mental e social), possam ter a satisfação das suas necessidades, na rotina diária e uma reintegração familiar e social (Faro, 2006).

\section{A IMPORTÂNCIA DOS ASPECTOS PSICOLÓGICOS NA REABILITAÇÃO}

Atualmente não se pode mais separar os componentes mente e corpo, como eram vistos no passado, pois ao longo dos anos, novos estudos foram realizados e descobertos que os aspectos psicológicos exercem influência sobre os aspectos orgânicos, portanto, não se pode falar de um sem citar o outro, pois toda doença física interfere no estado emocional do indivíduo (Simonetti, 2011).

A doença também traz os seus ganhos secundários, ou seja, ela não é feita somente de perdas. No processo de adoecimento, o paciente pode ganhar mais atenção e cuidados, deixar de exercer suas atividades diárias, como trabalhar, pode sentir pena de si mesmo e conseguir um pretexto para explicar dificuldades em diversas situações de sua vida, como existenciais, profissionais ou amorosas. Esses ganhos secundários da doença evidenciam o quanto os aspectos psicológicos podem 
atuar como fatores que contribuem para manter o indivíduo na situação de adoecimento, e a não investir no processo de reabilitação (Simonetti, 2011).

As emoções também são processos constituídos de forma subjetiva, envolvendo dimensões biológicas, individuais, psíquicas, sociais e culturais. Existem várias formas de expressão das emoções. Quando uma pessoa intensifica uma determinada forma de emoção ou quando ela a reprimi, pode gerar consequências para a sua saúde, causando, por exemplo, estresse (Bonomo \& Araujo, 2009).

Em alguns casos, existe associação entre doenças coronarianas e estados de ansiedade, raiva e depressão. Tais estados podem ter impacto desfavorável no prognóstico desta doença, podendo influenciar em comportamentos prejudiciais à saúde, como na diminuição da qualidade do sono, má alimentação, obesidade, sedentarismo, tabagismo, consumo de álcool e drogas, e até podendo provocar isolamento social (Bonomo \& Araujo, 2009).

O humor irritável e ansioso pode ocasionar arritmias devido à intensificação da atividade simpática (aumento da atividade cardíaca). Existe uma relação entre humor irritável e a ocorrência de infartos não fatais do miocárdio, e nas duas horas consecutivas a eventos de raiva, aumenta em dobro o risco de infarto do miocárdio. Sendo assim, relações afetivas saudáveis e humor eutímico (humor normal) podem ter influências no melhor enfrentamento da doença (Bonomo \& Araujo, 2007). Portanto, o estado psicológico do paciente deve ser considerado na reabilitação, pois interfere no desenvolvimento do prognóstico e na adesão ao tratamento (Grifo nosso).

A adesão ao tratamento do paciente com doença arterial coronariana refere-se ao processo de aceitação e adaptação quando este resolve seguir as recomendações da equipe de saúde, aderindo às medidas necessárias que contribuam para a sua reabilitação (Abreu-Rodrigues \& Seidl, 2008). Por esses motivos, as informações transmitidas pela equipe de saúde aos pacientes e às pessoas que o acompanham prestando apoio social, devem ser utilizadas numa linguagem compreensível, facilitando na adesão ao tratamento, pois isso auxilia no processo de reabilitação e na promoção da saúde do paciente (Mansur et al., 2001).

Quando o paciente não possui conhecimento sobre as condições que favorecem o surgimento e o desenvolvimento da sua doença, pode levar à adoção de medidas de prevenção e controle inadequadas (Gama et al., 2012). 
Estudos também mostram que o suporte social pode reduzir o risco da doença se agravar ao promover comportamentos relevantes para uma melhor reabilitação, que resulte em um melhor funcionamento cognitivo, psicológico e físico do paciente. A presença de suporte social ajuda na adesão ao tratamento e na melhora do prognóstico do paciente cardíaco, decorrente do fato de amenizar os agravos secundários gerados pela doença, como a depressão, o estresse pós-traumático e a ansiedade. $\mathrm{O}$ enfrentamento de uma doença crônica é dificultado quando o indivíduo se sente sozinho e/ou excluído socialmente (Abreu-Rodrigues \& Seidl, 2008).

A cirurgia cardíaca também gera, inevitavelmente, insegurança e ansiedade. Fantasias em relação à operação são comuns em pacientes cardiopatas. Algumas ansiedades como abrir o peito, o medo do CTI (Centro de Terapia Intensiva) e da morte são muito presentes. Todas essas ansiedades deixam o paciente vulnerável diante da limitação e da morte, sua identidade fica abalada diante da perda do controle de si mesmo. Quanto mais vulnerável o paciente se sentir, mais ansioso e deprimido ficará e uma série de fantasias e mecanismos de defesa serão acionados, como uma forma de manter o equilíbrio fisiológico e psicológico (Côrte, 1998).

Identificar como o paciente enfrenta e lida com a situação de estar aguardando uma cirurgia, é de suma importância não só para o psicólogo, mas também para os profissionais que the acompanham no hospital (Vargas et al., 2006).

Existe uma ansiedade considerada desejável, que impulsionará o paciente a agir, fazendo perguntas à equipe, relacionando-se com os familiares e aceitando o tratamento e as restrições impostas pelo preparo cirúrgico. No entanto, um alto grau de ansiedade, pode fazer o paciente se tornar apático, o que ocasionará dificuldades para compreender as orientações da equipe. Já o baixo grau de ansiedade, pode ocasionar introversão, tendo como consequência a resistência em compreender a situação vivida (Vargas et al., 2006).

Dos diagnósticos feitos no pré e no pós-operatório, o de ansiedade é um dos mais comuns. É um fenômeno vivenciado por quase todos os pacientes cirúrgicos. A ansiedade pode influenciar na resposta do paciente frente ao tratamento cirúrgico e acarretar efeitos negativos sobre a recuperação pós-operatória e na reabilitação (Vargas et al., 2006).

Sendo assim, é fundamental que no pré-operatório o paciente possa falar de suas fantasias e angústias, que seja orientado com todas as informações possíveis Rev. Psicol Saúde e Debate. Ago., 2021:7(2): 131-150. 
sobre os procedimentos da cirurgia, suas etapas, as consequências, o despertar no $\mathrm{CTI}$, como ficará nesse momento e as sensações que possivelmente poderão surgir. Também é importante que a equipe cirúrgica seja informada dos temores do paciente e os fatores que os desencadearam (Côrte, 1998).

O acompanhamento no pós-operatório que também envolve toda a equipe proporciona ao paciente a sensação de estar protegido e sendo cuidado, em um dos momentos mais difíceis do tratamento. O impacto emocional que o paciente vivencia diante da situação de estar cercado por tubos e aparelhos, a imobilidade, a dor, o desconforto, as restrições e a privação do contato com os familiares, leva o paciente a um sentimento de desproteção e abandono (Côrte, 1998).

Dependendo do ritmo que o paciente está habituado, as restrições pós-cirúrgicas podem promover reações variadas. Há aqueles que não conseguem ficar parados por serem muito ativos, irritam-se com facilidade, negam a fragilidade do seu estado, mostrando-se fortes e prontos para retomarem suas atividades cotidianas. Outros são mais passivos e retraídos, sendo excessivamente dependentes, se utilizando dos “ganhos da doença” para exigir atenção especial (Côrte, 1998).

Da saída do CTI para a enfermaria, até a alta hospitalar, o paciente vive os principais momentos no processo de resgate da autoconfiança. Portanto, o encorajamento para retomar suas atividades diárias, incentivando para que se alimente e seja higiênico, bem como, o orientando quanto ao que deve ser evitado, o que está liberado e como será sua rotina em casa, ajudam na recuperação do controle sobre si mesmo e a se sentir amparado até que esteja pronto para ser independente novamente (Côrte, 1998).

Após a realização da cirurgia, vem a fase da readaptação, e muitos podem sentir dificuldades nesse momento. O sentimento expressado pelos pacientes nesta fase geralmente é a insônia, pois após a cirurgia necessitam dormir numa posição específica (barriga para cima) à qual não estavam acostumados, e essa privação de sono provoca alterações no organismo. Também apresentam preocupações relacionadas à nova rotina que pode desencadear estado de tristeza, momentos de baixa autoestima e podendo até mesmo levar ao estado de depressão (Galter et al., 2010).

Outros aspectos que sofrem modificações são os hábitos alimentares, essas mudanças podem ocasionar estresse, pois os pacientes se sentem reprimidos por não 
poder comer certos alimentos que gostam, e sabem que infringir a dieta podem sofrer danos (Galter et al., 2010).

A doença arterial coronariana também interfere na atividade sexual dos pacientes, estando relacionada ao medo de complicações cardíacas durante 0 ato sexual. A intolerância ao esforço físico devido à dor no peito faz com que muitos pacientes não retornem à atividade sexual após a cirurgia, influenciando diretamente na sua qualidade de vida (Galter et al., 2010).

No período de reabilitação muitos sentimentos surgem nos pacientes com doença arterial coronariana, como o sentimento de dependência, devido ter que precisar de outras pessoas para executar as atividades do cotidiano, e quando não podem realizar as atividades da mesma forma que realizavam antes, sentem-se desvalorizados e insatisfeitos, se configurando como uma perda da autonomia, podendo provocar conflitos e mudanças na percepção de seu papel dentro do contexto familiar, profissional e social (Galter et al., 2010).

Mas os sentimentos mais presentes na fase da reabilitação são o medo, a ansiedade e a dependência. As dificuldades encontradas em relação às limitações físicas e modificações nos comportamentos podem ser descritas como "perdas": perda da independência, perda do prazer da alimentação, perda do interesse sexual etc. (Galter et al., 2010).

Como afirma Simonetti:

Uma situação de perdas, é como poderia ser definida a doença, afinal, perde-se a saúde, perde-se a autonomia, perde-se tempo e dinheiro, e muitas outras coisas, isso quando não se perde mesmo a própria vida. Tantas perdas, muitas delas reais e outras tantas imaginárias, abrem uma espécie de "caixa de pandora" de consequências subjetivas para a pessoa adoentada. $O$ ser humano comumente confere sentido a tudo o que ele vivencia, e com o adoecimento não é diferente. $O$ conjunto de sentidos que 0 sujeito confere a sua doença constitui, como consequência, o campo dos aspectos psicológicos (Simonetti, 2011, pp. 17-18).

$\mathrm{O}$ apoio emocional dos familiares aos pacientes com doença arterial coronariana é muito importante, pois ajudará para a reabilitação se tornar mais efetiva, assim o paciente se sentirá acolhido e respeitado, gerando uma maior aceitação pelas mudanças que serão necessárias nesse processo (Galter et al., 2010). A família representa o espaço de socialização, de promoção de estratégias coletivas, possibilitando o desenvolvimento individual e grupal de seus membros. Sua dinâmica é Rev. Psicol Saúde e Debate. Ago., 2021:7(2): 131-150. 
diferenciada pelo seu próprio desenvolvimento e pelas políticas econômicas e sociais (Dias, 2011).

Quando ocorre a descoberta de uma doença cardíaca como a doença arterial coronariana, esta família acaba tendo que exigir de si novos arranjos, e passa a se ajustar à rotina de cuidados diários com o paciente, desde o período de hospitalização, alta e retorno ao lar (Dantas et al., 2002).

O percurso do adoecimento, principalmente na fase aguda desta doença, faz com que estes ajustamentos da família sejam necessários, por isso os familiares muitas vezes adquirem habilidades de coping (conjunto de estratégias para se adaptar às situações adversas). Estas estratégias são importantes para manejar o processo de recuperação do paciente quando este recebe alta do hospital e volta para casa, sendo elas: procurar ajuda especializada quando necessário, aprender a lidar com as suas preocupações e ansiedades, saber buscar informações necessárias para enfrentar os problemas apresentados etc. (Dantas et al., 2002).

Muitas vezes se torna difícil para os profissionais de saúde perceber de que forma eles poderão contar com a ajuda desses familiares para a adesão do paciente ao tratamento e no momento que o paciente recebe alta. A tarefa dos profissionais deve ser então de orientar e dar os subsídios necessários para que a família possa realizar esses cuidados longe da rotina do hospital (Dantas et al., 2002).

Portanto, é necessário que seja estabelecido um diálogo entre toda a equipe de saúde e a família do paciente, e para que esse vínculo seja criado é preciso que todos estejam envolvidos para dar o apoio e os cuidados necessários, disponibilizando atendimento digno, respeitando o paciente e toda a sua família (Schneider et al., 2008).

\section{O PAPEL DO PSICÓLOGO NO PROCESSO DE REABILITAÇÃO}

Compreendida como um processo, a reabilitação contempla múltiplas dimensões, indo além da recuperação de funções perdidas ou alteradas. Além disto, é importante ressaltar que reabilitar não significa curar e nem fragmentar o indivíduo. "Reabilita-se a pessoa, o ser humano na sua magnitude física, emocional e social" (Faro, 2006, p. 129). Conforme Angerami (2004, p. 106):

Reabilitação é a ação coordenada e contínua de uma equipe de técnicos competentes junto ao paciente, com o objetivo de auxiliálo a realizar suas potencialidades físicas, sociais, psíquicas e laborais, de modo a alcançar um melhor controle sobre si mesmo e sobre seu ambiente, enfrentando a realidade da vida.

Rev. Psicol Saúde e Debate. Ago., 2021:7(2): 131-150. 
O paciente em reabilitação deve ser visto como um ser integral, cuja equipe multiprofissional deve conhecer as razões dos seus comportamentos para melhor ajudá-lo, e assim este paciente terá melhor aproveitamento em seu processo de reabilitação (Oliveira, 2000).

Na reabilitação, o paciente possui papel de extrema relevância, uma vez que ele é o foco de intervenção da equipe, é quem direciona e determina os resultados de seu próprio processo. A família quando incluída no processo de reabilitação possui papel dinâmico no tratamento, através do aprendizado dos cuidados necessários ao paciente (Leite \& Faro, 2005).

O momento em que o paciente, a família e o profissional se deparam com as incapacidades decorrentes da doença, se caracteriza como a fase mais difícil de ser enfrentada. Esta etapa necessita de ajustes e adaptações, frente às reações emocionais que podem surgir diante das alterações físicas ou mentais sofridas (Faro, 2006).

É necessário que o psicólogo esteja sempre em contato com a equipe multiprofissional, dando contribuições provenientes do seu saber psicológico, e através de instrumentos como a escuta psicológica, possibilitar novas formas de lidar com aquele paciente e os familiares, a partir disto o seu trabalho pode ser reconhecido como importante para aquela equipe de reabilitação (Fossi \& Guareschi, 2004).

Araújo (2007) destaca que a visão contemporânea de reabilitação, está fundamentada em quatro preceitos básicos descritos a seguir: Intervenção centrada no paciente; Ênfase no processo (interação, negociação, comunicação, educação e troca de informações); Atuação fundamentada em um modelo que integra aspectos de autocuidado, produtividade, recreação e socialização, resultantes dos componentes físico, sociocultural, mental/emocional e filosófico/espiritual; Responsabilização do paciente na resolução de problemas, estabelecimento de planos, incluindo até a orientação do meio social na execução dos cuidados.

Araújo (2007) também destaca que dentre os pontos relevantes a serem considerados no processo de reabilitação estão: A participação do paciente no processo decisório; Identificação dos fatores associados aos modos de participação do paciente; Caracterização das percepções e expectativas de pacientes e profissionais em relação à participação ideal; Qualidade da relação paciente-profissional; Estrutura 
do atendimento; Aspectos relacionados à limitação física e comportamentos ou características pessoais do paciente.

É necessário que este profissional atenda também a família do paciente para saber quais as demandas que esta lhe traz, as dúvidas, questionamentos e as suas percepções a respeito do adoecimento do paciente. Este profissional dará apoio, acolhimento para o paciente e sua família e trabalhará em conjunto com a equipe multiprofissional para favorecer um atendimento de qualidade, promovendo o respeito aos indivíduos envolvidos para que possam confiar nos serviços oferecidos pela instituição e se sintam acolhidos (Cantarelli, 2009).

O psicólogo passa a ser o intermediário entre a equipe e o paciente, pois possui o conhecimento necessário para compreender os aspectos emocionais que estão envolvidos na reabilitação do paciente. Portanto, a responsabilidade do psicólogo na reabilitação consiste em compartilhar com a equipe de profissionais, os aspectos psicológicos da personalidade dos pacientes que os levam a ter certas atitudes e comportamentos, porém avaliando o que pode e deve ser compartilhado com a equipe e o que não pode, pois existem situações que o psicólogo deve guardar sigilo (Angerami, 2004).

\section{CONSIDERAÇÕES FINAIS}

O paciente com doença arterial coronariana enfrenta dificuldades desde a descoberta do diagnóstico. Ao receber este diagnóstico, sofre um impacto psicológico, sendo difícil aceitar que apresenta uma doença crônica, que precisará passar por um tratamento invasivo e possivelmente pela cirurgia, que pode provocar sentimentos de ansiedade e medo, ficando na expectativa se este procedimento será efetuado com sucesso ou se culminará no fracasso.

Depois de realizada a cirurgia, o paciente passa por um período de recuperação, e este momento é o mais delicado, pois poderá ter restrições nos âmbitos da alimentação, nas atividades que antes realizava, dificuldades no ato sexual etc. Essas mudanças são experienciadas com temor e ansiedade dificultando na adesão ao tratamento.

Pela doença arterial coronariana trazer sofrimento e comprometimento na qualidade de vida destes pacientes, se faz necessária à atuação do psicólogo para promover a reabilitação, porém a reabilitação deve ser realizada em conjunto com uma 
equipe multiprofissional, cada profissional com seu campo de saber contribuindo na melhora do quadro do paciente e podendo trocar informações entre si para ajudar no processo de adoecimento do paciente.

O psicólogo é o profissional que irá dar escuta e suporte emocional, tendo conhecimentos para observar as emoções e os sentimentos que interferem no processo de adoecimento, na adesão ao tratamento e na reabilitação do paciente.

Não podemos deixar de ressaltar a dificuldade em encontrar material sobre a reabilitação e o papel do psicólogo neste processo junto aos pacientes com doença arterial coronariana, isso mostra a necessidade de implantação de programas de reabilitação nas unidades cardiológicas com a inserção do psicólogo na equipe multiprofissional e o desenvolvimento de futuros trabalhos sobre este tema que é tão relevante para a área da saúde de todos os profissionais envolvidos.

Para concluirmos, foi possível observar a importância dos aspectos psicológicos na reabilitação do paciente com doença arterial coronariana. A forma como este vai enfrentar todas as mudanças que ocorrem no processo de reabilitação e no seu estado emocional podem influenciar na sua adesão ao tratamento. A forma como percebe a si mesmo, a relação com a sua família e com a equipe de saúde influencia em todo o processo. Assim, é necessário compreender esse paciente como um ser único, que possui sentimentos, emoções, desejos e vontades que precisam ser respeitadas em sua singularidade e o não menos importante: que ele tenha o atendimento do psicólogo hospitalar.

\section{REFERÊNCIAS}

Abreu-Rodrigues, M., \& Seidl, E. M. F. (2008). A importância do apoio social em pacientes coronarianos. Paidéia, 18(40), 279-288. Recuperado de: https://www.scielo.br/scielo.php?script=sci_arttext\&pid=S0103-

863X2008000200006\#: :text=Observa\%2Dse\%20que\%20a\%20associa\%C3\%A7 \%C3\%A3o,\%2Dtraum\%C3\%A1tico\%20e\%20ansiedade\%20(Pedersen\%2C

Angerami, W. A. (2004). Tendências em psicologia hospitalar. São Paulo: Pioneira Thomson Learning.

Araujo, T. C. C. F. (2007). Psicologia da reabilitação: Pesquisa aplicada à intervenção hospitalar. Revista da SBPH, 10(2), 63-72. Recuperado de: http://pepsic.bvsalud.org/scielo.php?script=sci_arttext\&pid=S15160858200700020000

Bonomo, A. M. S., \& Araujo, T. C. C. (2009). Psicologia aplicada à cardiologia: um estudo sobre emoções relatadas em exame de holter. Psicologia: Teoria e 
$\begin{array}{lrl}\text { Pesquisa, } & \text { 25(1), } & \text { 65-74 }\end{array}$

Recuperado

de:

https://www.scielo.br/scielo.php?pid=S0102-

37722009000100008\&script=sci_abstract\&tlng=pt

Cantarelli, A. P. S. (2009). Novas abordagens da atuação do psicólogo no contexto hospitalar. Revista SBPH, 12(2), 137-147. Recuperado de: http://pepsic.bvsalud.org/scielo.php?script=sci_arttext\&pid=S1516-

08582009000200011

Cantus, D. S., \& Ruiz, M. D. C. S. (2011). A cardiopatia isquêmica na mulher. Revista Latino Americana de Enfermagem, 19(6), 1-8. Recuperado de: https://www.scielo.br/scielo.php?script=sci_arttext\&pid=S0104$11692011000600025 \&$ lng=en\&nrm=iso\&tlng=pt

Côrte, R. L. (1998). Importância do aspecto psicológico na reabilitação do paciente cardiopata. Revista SOCERJ, 11(1), 31-37. Recuperado de: http://sociedades.cardiol.br/socerj/revista/1998/1998_02.pdf

Dantas, R. A. S., Stuchi, R. A. G., \& Rossi, L. A. (2002). A alta hospitalar para familiares de pacientes com doença arterial coronariana. Revista da Escola de Enfermagem da USP, 36(4), 345-350. Recuperado de: https://www.scielo.br/scielo.php?script=sci_arttext\&pid=S008062342002000400008

Dias, M. O. (2011). Um olhar sobre a família na perspectiva sistêmica o processo de comunicação no sistema familiar. Revista Gestão e Desenvolvimento, v.19, 139156. Recuperado de: https://repositorio.ucp.pt/handle/10400.14/9176

Eickhoff, P. C., Schneider, N., \& Aozani, J. P. (2014). O trabalho do psicólogo com pacientes em reabilitação. XV jornada de extensão da Universidade do Noroeste do Estado do Rio Grande do Sul. ljuí: Unijuí. Recuperado de: https://www.publicacoeseventos.unijui.edu.br/index.php/salaoconhecimento/article/v iew/3371/2779

Escudeiro, G., \& Melo, W. A. (2010). Prevalência dos fatores de risco em pacientes submetidos à angioplastia coronariana. V amostra interna de trabalhos de iniciação científica do centro universitário de Maringá. Paraná: CESUMAR. Recuperado de: http://www.cesumar.br/prppge/pesquisa/mostras/quin_mostra/gisele_escudeiro_3.p df

Faro, A. C. M. (2006). Enfermagem em reabilitação: ampliando os horizontes, legitimando o saber. Revista da Escola de Enfermagem da USP, 40(1), 128-133. Recuperado de: https://www.scielo.br/scielo.php?pid=S008062342006000100019\&script=sci_abstract\&tlng=pt

Fossi, L. B., \& Guareschi, N. M. F. (2004). A psicologia hospitalar e as equipes multidisciplinares. Revista da SBPH, 7(1), 29-43. Recuperado de: http://pepsic.bvsalud.org/scielo.php?script=sci_arttext\&pid=S1516-

08582004000100004 
Galter, C., Rodrigues, G. C., \& Galvão, E. C. F. (2010). A percepção do paciente cardiopata para vida ativa após recuperação de cirurgia cardíaca. Journal of the Health Sciences Institute, 28(3), 255-258. Recuperado de: http://repositorio.unip.br/wp-content/uploads/2020/12/V28_n3_2010_p255-258.pdf

Gama, G. G. G., Mussi, F. C., Pires, C. G. S., \& Guimarães, A. C. (2012). Crenças e comportamentos de pessoas com doença arterial coronária. Ciência \& Saúde Coletiva, 17(12), 3371-3383. Recuperado de: https://www.scielo.br/scielo.php?script=sci_arttext\&pid=S141381232012001200022

Gus, A., Fischmann, I., \& Medina, C. (2002). Prevalência dos fatores de risco da doença arterial coronariana no Estado do Rio Grande do Sul. Arquivos Brasileiros de Cardiologia, 78(5), 478-483. Recuperado de: http://publicacoes.cardiol.br/abc/2002/7805/78050005.pdf

Jurkiewicz, R., \& Romano, B. W. (2009). Doença arterial coronariana e vivência de perdas. Arquivos Brasileiros de Cardiologia, 93(4), 352-359. Recuperado de: https://www.scielo.br/scielo.php?pid=S0066-

782X2009001000007\&script=sci_abstract\&tlng=pt

Lamas, A. R. (2010). Fatores de risco modificáveis para a doença arterial coronariana prevalentes nos trabalhadores de enfermagem. Dissertação de Mestrado, Universidade Federal do Estado do Rio de Janeiro. Rio de Janeiro: UFRJ. Recuperado de: http://www.repositoriobc.unirio.br:8080/xmlui/bitstream/handle/unirio/12180/Disserta\%c3\%a7\%c3\%a3o$\% 20$ Alinny\%20Rodrigues\%20Lamas.pdf?sequence=1\&isAllowed=y

Leite, V. B. E., \& Faro, A. C. M. (2005). O cuidar do enfermeiro especialista em reabilitação físico-motora. Revista da Escola de Enfermagem da USP, 39(1), 92-96. Recuperado de: https://www.scielo.br/scielo.php?script=sci_arttext\&pid=S008062342005000100012

Maia, C. O., Goldmeier, S., Moraes, M. A., Boaz, M. R., \& Azzolin, K. (2007). Fatores de risco modificáveis para doença arterial coronariana nos trabalhadores de enfermagem. Acta Paulista de Enfermagem, 20(2), 138-142. Recuperado de: https://www.scielo.br/scielo.php?script=sci_arttext\&pid=S0103-

21002007000200005\#: :text=Entre\%20os\%20modific\%C3\%A1veis\%20est\%C3\%A 30\%20a,a\%20HAS\%20e\%20a\%20obesidade.

Mansur, A. P., Mattar, A. P. L., Tsubo, C. E., Simão, D. T., Yoshi, F. R., \& Daci, K. (2001). Prescrição e aderência das estatinas em pacientes com doença arterial coronariana e hipercolesterolemia. Arquivos Brasileiros de Cardiologia, 76(2), 111114. Recuperado de: http://publicacoes.cardiol.br/abc/2001/7602/7602002.pdf

Oliveira, B. S., Castro, Y. T. B. O., Silva, L. D. C., Azevedo, P. R., \& Silva, A. C. O. (2015). Cotidiano de mulheres com doença arterial coronariana: uma perspectiva de gênero. Revista Baiana de Enfermagem, 30(1), 305-315. Recuperado de: https://aratuipe.ufba.br/index.php/enfermagem/article/viewFile/14591/pdf_29 
Oliveira, R. A. (2000). Elementos psicoterapêuticos na reabilitação dos sujeitos com incapacidades físicas adquiridas. Análise Psicológica, 4(18), 437-453. Recuperado de: http://publicacoes.ispa.pt/index.php/ap/article/view/391/pdf

Pêgo-Fernandes, P. M., Gaiotto, F. A., \& Guimarães-Fernandes, F. (2008). Estado atual da cirurgia de revascularização do miocárdio. Revista de Medicina, 87(2), 9298.

Romano, B.W. (1998). A Prática da Psicologia nos Hospitais. (2a ed.). São Paulo: Pioneira.

Simonetti, A. (2011). Manual de Psicologia Hospitalar: o mapa da doença. (6a ed.). São Paulo: Casa do Psicólogo

Sociedade de Cardiologia do Estado do Rio de Janeiro (SOCERJ). Doença coronariana. (2013). Recuperado de: https://socerj.org.br/areapublico/?p=291\#: :text=A\%20doen\%C3\%A7a\%20coronariana\%20\%C3\%A9\%20o,i mpedir\%20a\%20passagem\%20do\%20sangue.

Schneider, D. G., Manschein, A. M. M., Ausen, M. A. B., Martins, J. J., \& Albuquerque, G. L. (2008). Acolhimento ao paciente e família na unidade coronariana. Texto \& Contexto Enfermagem, 17(1), 81-89. Recuperado de: https://www.scielo.br/scielo.php?pid=S010407072008000100009\&script=sci_abstract\&tIng=pt

Vargas, T. V., Maia, E. M., \& Dantas, R. A. S. (2006). Sentimentos de pacientes no préoperatório de cirurgia cardíaca. Revista Latino-Americana de Enfermagem, 14(3), 1-6. Recuperado de: https://www.scielo.br/scielo.php?pid=S0104$11692006000300012 \&$ script=sci_abstract\&tlng=pt 